\title{
Multiple sclerosis: looking beyond autoimmunity
}

\author{
Abhijit Chaudhuri PhD FRCP ${ }^{1}$ Peter O Behan DSc FRCP2
}

J R Soc Med 2005;98:303-306

'My dictionary gives the Latin root for falsity as fallere, which is the same root for the word failure.'-Lewis Thomas ${ }^{1}$

The chronic incurable disorder multiple sclerosis (MS) is characterized by neurodegeneration, multifocal demyelination and astroglial proliferation (gliosis). ${ }^{2}$ The prevalence of MS is influenced by geography and genetics. In the Western world it is a leading cause of neurological disability in the young. The cause and exact pathogenesis are still unknown. Some see MS as a T-cell-driven autoimmune inflammatory disease, targeting the myelin sheaths in the central nervous system, ${ }^{3}$ but there is no proof. Unlike autoimmune conditions such as rheumatoid arthritis, systemic lupus erythematosus or myasthenia gravis, MS has no specific immunological marker. ${ }^{2,4}$

An animal model that has been used in MS research is experimental allergic encephalomyelitis (EAE), in which demyelination is induced by sensitization against myelin basic protein. Clinically and pathologically, however, EAE resembles acute disseminated encephalomyelitis (ADEM) rather than $\mathrm{MS}^{2}{ }^{2}$ Nonetheless, the EAE model has been used to drive the autoimmune theory and to develop treatments. An inflammatory hypothesis of demyelination also fails to explain various salient features of the disease (Box 1). ${ }^{5-7}$ Here, we explain our view that research and treatment strategy in MS need to change direction.

\section{HISTORICAL PERSPECTIVE}

It was during the 1940s and 1950s that researchers became interested in experimentally induced demyelination. Hyperacute and acute demyelination (ADEM) in man came to be recognized as a complication of immunization with brain derived tissue (e.g. post-rabies-vaccine encephalomyelitis) and a similar 'allergic' basis of demyelination in MS was postulated. Adams made a detailed histological comparison of ADEM and MS in terms of morphological criteria for demyelinative disease-namely, destruction of myelin sheaths of nerve fibres; relative sparing of all other elements of nervous tissue (i.e. nerve cells, axis cylinders

${ }^{1}$ Department of Neurology, Essex Centre for Regional Neurosciences, Romford RM7 OBE; '2University of Glasgow, Scotland, UK

Correspondence to: Dr A Chaudhuri

E-mail: abhijitchaudhuri@btinternet.com and supporting structures); and distribution of lesions, often perivenous, in multiple locations throughout the brain and spinal cord or to single foci spreading from one or more centres. ${ }^{8}$ From Table 1 , summarizing his observations, it is apparent that there are similarities but also important differences. Adams regarded the syndrome of acute bilateral optic neuritis and transverse myelitis (neuromyelitis optica, Devic's disease) as a regional variant of ADEM; the clinical syndrome of myelitis was judged merely a matter of localization within the spinal cord, where the tight confines of the pia and the oedema of very rapidly evolving lesions of ADEM led to infarction-necrosis (a condition seldom seen in the brain).

Subsequently, one of us (POB) showed that both EAE and ADEM are T-cell-dependent, organ-specific, autoimmune diseases of the central nervous system. ${ }^{9,10}$ This has not been found true for MS, despite three decades of intensive research. Indirect evidence cited in support of an autoimmune pathogenesis for MS has likewise been found wanting (Table 2).

\section{DISSOCIATION OF MS FROM EAE AND ADEM}

The clinical, radiological, and histological differences between EAE and MS, we believe, argue against a common pathogenesis. EAE is typically a monophasic disorder, and even subacute or chronic relapsing models of EAE represent

Box 1 Important facts about MS that cannot be explained by the concept of myelin-specific autoimmunity

- Age effect of migration

- Geographic variation (higher prevalence in most northern latitudes)

- Maternal contribution to disease risk (Ref. 5)

- Early and extensive grey matter involvement (estimated number of deep grey matter lesions per gram wet weight is higher than in any other brain structure [Ref. 6])

- Progressive brain and spinal cord atrophy, beginning at the stage of clinically isolated demyelinating syndromes (Ref. 7)

- Selective anatomical localization, symmetry and sharp margins of plaques

- Absence of specific immunological marker

- Effect of stress

- General failure of immunotherapies that are highly successful in other organ-specific autoimmune diseases and transplant rejection

- Associations with Charcot-Marie-Tooth disease and neurofibromatosis-1 (Ref. 2) 
Table 1 Comparative pathology of the demyelinative lesions in acute disseminated encephalomyelitis (ADEM) and multiple sclerosis (MS)

\begin{tabular}{|c|c|c|}
\hline Pathological characteristics & ADEM & Acute and chronic MS \\
\hline Distribution & Focal, regional or diffuse & $\begin{array}{l}\text { Multifocal; as a rule entire central nervous system is affected } \\
\text { in chronic MS }\end{array}$ \\
\hline Age of lesions & Always same and uniform & Always of different ages (both in acute and chronic MS) \\
\hline Size of lesions & $0.1-1.0 \mathrm{~mm}$ & $1.0 \mathrm{~mm}$ or less to $5+\mathrm{cm}$ \\
\hline \multicolumn{3}{|l|}{ Relation of lesions to: } \\
\hline Veins & Always & Prominent and usual \\
\hline Pia & Usual & Rare \\
\hline \multicolumn{3}{|l|}{ Degeneration of: } \\
\hline Axis cylinders & Mild to severe & Mild to moderate \\
\hline Nerve cells & Mild & Mild to moderate \\
\hline Oligodendroglia & Restricted to lesions & Restricted to lesions \\
\hline Astrocytosis & Mild & Marked in chronic MS \\
\hline Tissue necrosis & May be severe & Rarely severe \\
\hline Perivascular infiltration & Always and marked & Usually (but not always) present; prominent only in acute MS \\
\hline Meningeal inflammation & Minimal to pronounced & None \\
\hline $\begin{array}{l}\text { Vascular damage and fibrin } \\
\text { deposition }\end{array}$ & $\begin{array}{l}\text { Constant and severe in } \\
\text { hyperacute cases }\end{array}$ & Mild or none \\
\hline Microglial proliferation & Marked, with pleomorphic forms & Pleomorphic forms in acute cases \\
\hline
\end{tabular}

Table 2 Autoimmunity and multiple sclerosis (MS)

\section{Evidence used to support the concept of autoimmunity in MS}

Predominance of women affected, as in rheumatoid arthritis and systemic lupus erythematosus

Association of other autoimmune diseases in affected individuals and families

Association with HLA haplotypes

Antimyelin antibodies in serum

Oligoclonal bands in cerebrospinal fluid

Specific T-cell response identified during MS relapses

Partial therapeutic response of relapses to beta-interferons, glatiramer and other immunotherapies

\section{Comments}

Predominance of women also seen in non-autoimmune disorders such as migraine

Not proven in large epidemiological studies of MS

The strongest HLA links are observed with hereditary

haemochromatosis and narcolepsy, metabolic and neurodegenerative diseases respectively

Antimyelin antibodies can be detected in neurological disorders other than MS, and some antimyelin antibodies have a reparative function

These bands are reported in other neurological diseases such as subacute sclerosing panencephalitis and neurosyphilis, conditions that are not autoimmune. Oligoclonal-band-negative cases of MS have been reported

No T-cell response is unique or specific in MS

Relapse prevention in MS may not translate into disability prevention. No immunotherapy has reduced long-term disability in MS recurrent challenges to some encephalitogenic antigen, a phenomenon that has not been shown to apply in MS. The progressive and global brain and spinal cord atrophy that characterizes the human disease from its earliest stages ${ }^{7}$ has not been reproduced in animals with EAE. EAE and ADEM also differ from MS in that the uninvolved white matter is normal. ${ }^{11}$
One of the characteristic histological features of EAE is destruction of cerebral endothelial cells by an immunological mechanism, and this is seen in human cases of ADEM after immunization (post-rabies vaccine encephalomyelitis) or endotoxic shock, ${ }^{12}$ but not in MS. Even from the early days it was acknowledged that chronic relapsing MS had pathological features that were absent in acute or subacute 
EAE, such as the large sizes and confluence of individual demyelinating lesions ('plaques'), the shadow plaques and the appearance of fresh lesions at the borders of the older ones 'as though the pathological process had spread in a succession of waves from a more central focus'. ${ }^{8}$ Furthermore, there are inherent pitfalls in the assumption of a common pathogenesis based on morphological similarities alone, when the range of histological responses to injury is so limited within the nervous system. For example, ischaemic brain tissue will look much the same in cerebral infarcts due to systemic lupus, cardioembolic stroke or thrombotic stroke, though the pathogenesis and treatment will differ. Morphological issues apart, an important reason for questioning the extrapolation of EAE to MS pathogenesis is the failure to identify an encephalitogenic marker specific for MS. In Devic's disease (a variant of ADEM), Lennon et al. have now found an antibody that localizes with laminin at the blood-brain barrier. ${ }^{13}$ We would recommend immunosuppression in Devic's disease but not in MS.

\section{BEYOND AUTOIMMUNITY IN MS}

The definition of MS as a T-cell-specific autoimmune demyelinative disease is in our view too narrow. First, nearly 60 years of EAE-based research yielded not a single MS-halting therapy. This in itself should be an important reason to consider a shift in research direction. Although the existing disease-modifying therapies reduce relapse rates in some patients by up to one-third there is little evidence that the common features of fatigue, pain, depression and cognitive decline are positively influenced. ${ }^{14}$ There is also a concern, theoretical at present, that early benefit of reduced relapse rates may later be offset by accelerated brain atrophy. ${ }^{15}$

Second, neurodegeneration is now regarded as an important component in MS. It is neurodegeneration rather than demyelination that contributes to long-term disability. Several pathological and MRI studies indicate that grey matter involvement is early and extensive. Axonal transection may be degenerative, inflammatory or both, but brain and spinal cord atrophy is considered to be the direct result of neurodegeneration. If we accept axonal degeneration and neuronal loss to be essential features, then MS no longer fulfils the original criteria of a primary demyelinative disease and comparison with EAE becomes even less apposite. Longitudinal studies of brain volume in MS teach us that the rate of brain atrophy is independent of the disease subtype ${ }^{16}$ in other words, whether the disease is classed as relapsing-remitting or progressive, the loss of brain volume is the same and is due to neurodegeneration. While the concept of clinical and pathogenic heterogeneity ${ }^{17}$ has been proposed to identify an inflammatory subgroup in necropsy studies, it is clear that all patients, whatever their clinical phenotype, require neuroprotection.

Third, the most neglected aspect of MS research is prevention, and we believe that this again is explained by the erroneous assumption of autoimmunity. Several potentially modifiable environmental factors are associated with the risk of developing MS. One is infectious mononucleosis due to Epstein-Barr virus (EBV), which emerged in a large case-control study of MS and virus infection among US women (Nurses' Health Study I and II) as the single most important risk factor. ${ }^{18}$ Currently, the search for an effective EBV vaccine is focused on prevention of lymphoproliferative disorders and nasopharyngeal carcinoma in susceptible populations, ${ }^{19}$ but such a vaccine has huge potential for reducing the risk of several other debilitating clinical syndromes including MS. Smoking is another modifiable risk factor; ${ }^{20}$ in female nurses there was a clear dose-response relation between cigarette consumption and MS, and in a general population the risk of MS was higher in smokers than in never-smokers. ${ }^{21}$ Clearly, smoking needs to be especially discouraged, from the time of diagnosis, in a patient with MS.

Finally, of all the environmental risk factors associated with MS, vitamin D seems the most easily modified. ${ }^{22}$ In a longitudinal follow-up study of over 90000 women, those taking vitamin D supplements had a $40 \%$ lower incidence of MS than those who did not. ${ }^{23} \mathrm{MS}$ is rare in the tropics and a direct relation between sunlight exposure and MS has been confirmed in epidemiological studies since 1960. ${ }^{24}$ Therefore an argument can be made for vitamin D supplementation to reduce the risk of MS in areas of high disease prevalence in the northern latitudes where solar exposure is inadequate for vitamin D synthesis throughout the year. ${ }^{22}$

\section{CONCLUSIONS}

After six decades of autoimmune and EAE-based research, the time has come for a change in direction. For basic scientists, the challenge is to develop a new animal model of MS that replicates both demyelination and neurodegeneration. Solvent-induced demyelination might be such a model, in view of the association between solvent exposure and MS risk. ${ }^{25,26}$ For clinicians the task is to evaluate existing neuroprotective treatments. Possible candidate agents for randomized trials in early MS are antioxidants such as coenzyme $\mathrm{Q}_{10},{ }^{27}$ omega-3-essential fatty acids, ${ }^{28}$ minocyclin, ${ }^{29}$ and cannabinoids, ${ }^{30}$ alone or in combination.

\section{REFERENCES}

1 Thomas L. Falsity and failure. In: Late Night Thoughts on Listening to Mahler's Ninth Symphony. New York: Penguin Books, 1995:113 
2 Behan PO, Chaudhuri A, Roep BO. The pathogenesis of multiple sclerosis revisited. J R Coll Physicians Edinb 2002;32:244-65

3 Weiner HL. Multiple sclerosis is an inflammatory, T-cell mediated autoimmune disease. Arch Neurol 2004;61:1613-15

4 Chaudhuri A, Behan PO. Multiple sclerosis is not an autoimmune disease. Arch Neurol 2004;61:1610-12

5 Ebers GC, Sandovnick AD, Dyment DA, Yee JML, Willer CJ, Risch N. Parent-of-origin effect in multiple sclerosis: observations in halfsiblings. Lancet 2004;363:1773-4

6 Lumsden C. The neuropathology of multiple sclerosis. In: Bruyn GW, Vinken P, eds. Handbook of Clinical Neurology. Amsterdam: Elsevier, 1970:217-309

7 Dalton CM, Chard CT, Davies GR, et al. Early development of multiple sclerosis is associated with progressive grey matter atrophy in patients presenting with clinically isolated syndromes. Brain 2004;127:1101-7

8 Adams RD. A comparison of the morphology of the human demyelinative diseases and experimental 'allergic' encephalomyelitis. In: Kies MW, Alvord EC Jr, eds. "Allergic" Encephalomyelitis. Washington: Charles C Thomas, 1959:183-209

9 Behan PO, Geshwind N, Lamarche JB, et al. Delayed hypersensitivity to encephalitogenic protein in disseminated encephalitis. Lancet 1968;ii: $1009-12$

10 Behan PO, Kies MW, Lisak RP, et al. Immunologic mechanisms in experimental encephalomyelitis in non-human primates. Arch Neurol 1973;29:4-9

11 Ingelse M, Salvi F, Iannucci G, et al. Magnetization transfer and diffusion tensor MR imaging of acute disseminated encephalomyelitis. Am J Neuroradiol 2002;23:267-72

12 Graham DI, Behan PO, More IAR. Brain damage complicating septic shock. Acute haemorrhagic leukoencephalitis as a complication of generalised Shwartzman reaction. J Neurol Neurosurgery Psychiatry 1979;42:19-28

13 Lennon VA, Wingerchuck DM, Kryzer TJ, et al. A serum autoantibody marker of neuromyelitis optica: distinction from multiple sclerosis. Lancet 2004;364:2106-12

14 Chaudhuri A, Behan PO. Treatment of multiple sclerosis beyond the NICE guideline. Quart J Med 2005;98:373-8

15 Chaudhuri A. Beta interferon, progressive multiple sclerosis and brain atrophy. Lancet Neurol 2005;4:208-9
16 Kalkers NF, Ameziane N, Boost JCJ, Minnebo A, Polman CH, Barkhof F. Longitudinal brain volume measurement in multiple sclerosis. Rate of brain atrophy is independent of the disease subtype. Arch Neurol 2002;59:1572-6

17 Lucchinetti C, Bruck W, Parisi J, Scheithauer B, Rodriguez M, Lassmann H. Heterogeneity of multiple sclerosis lesions: implications for pathogenesis of demyelination. Ann Neurol 2000;47:707-17

18 Hernan MA, Zhang SM, Lipworth L, Olek MJ, Ascherio A. Multiple sclerosis and age at infection with common viruses. Epidemiology 2001; 12:301-6

19 Macsween KF, Crawford DH. Epstein-Barr virus-recent advances. Lancet Infect Dis 2003;3:131-40

20 Hernan MA, Olek MJ, Ascherio A. Cigarette smoking and incidence of multiple sclerosis. Am J Epidemiol 2001;154:69-74

21 Riise T, Nortvedt M, Ascherio A. Smoking is a risk factor for multiple sclerosis. Neurology 2003;61:1122-4

22 Chaudhuri A. Why we should offer routine supplementation of vitamin $\mathrm{D}$ in pregnancy and childhood to prevent multiple sclerosis. Med Hypotheses 2005;64:608-18

23 Munger KL, Zhang SM, O'Reilly E, et al. Vitamin D intake and incidence of multiple sclerosis. Neurology 2004;13:62:60-5

24 Acheson ED, Bachrach CA, Wright FM. Some comments on the relationship of the distribution of multiple sclerosis to latitude, solar radiation and other variables. Acta Psychiatry Scand 1960; 147(suppl): 132-47

25 Landtblom A-M, Flodin U, Soderfeldt B, Wolfson C, Axelson O. Organic solvents and multiple sclerosis: a synthesis of the current evidence. Epidemiology 1996;7:429-33

26 Riise T, Moen BE, Kryvik KR. Organic solvents and the risk of multiple sclerosis. Epidemiology 2002;13:718-20

27 Shultz CW. Coenzyme Q10 in neurodegenerative diseases. Curr Med Chem 2003;19:1917-21

28 Calon F, Lim GP, Yang F, et al. Docosahexanoic acid protects from dendritic pathology in an Alzheimer's disease mouse model. Neuron 2004;43:633-34

29 Chen M, Ona VO, Li M, et al. Minocycline inhibits caspase-3 expression and delays mortality in a transgenic mouse model of Huntington's disease. Nature Med 2000;6:797-801

30 Baker D, Pryce G, Giovannoni G, et al. The therapeutic potential of cannabis. Lancet Neurol 2003;2:291-8 